\title{
Tools for Specifying and Executing Synchronized Multimedia Presentations
}

\author{
Gerold Blakowski, Jens Hübel, Ulrike Langrehr \\ University of Karlsruhe, Institute for Telematics \\ Zirkel 2, D-W7500 Karlsruhe, Germany \\ Phone: $(++49)$ (721) 608-3414, Email: blakowski@ira.uka.de
}

\begin{abstract}
Multimedia applications require the handling of synchronization between media streams. We present tools for creating, editing and presenting synchronized multimedia objects. Specifying synchronization is supported by the graphical Synchronization Editor and execution of multimedia presentations is performed by the Synchronizer.

Major topics cover requirements resulting from underlying distributed heterogeneous environments and the consideration of user interactions. The tools we have developed are not restricted to a fixed set of media, but support the inclusion of arbitrary user-defined media.

The Synchronization Editor and the Synchronizer are part of the MODE project, that is used for the handling of distributed multimedia objects in NESTOR, an authoring-learning environment in development at the Universities of Karlsruhe and Kaiserslautern in cooperation with the CEC Karlsruhe, Digital Equipment.
\end{abstract}

\section{Introduction}

The integration of time dependent media in applications requires the synchronization of media streams. In this paper we describe object-oriented tools that support the graphical specification and execution of synchronized multimedia presentations.

The developed components are the user-friendly graphical Synchronization Editor and the Synchronizer covering the requirements resulting from distribution of media objects in a heterogeneous environment. These requirements include the handling of error conditions as well as the support of alternative presentation forms and different presentation qualities for the heterogeneous platforms. Other important requirements met by the tools are the integration of any kind of user-defined media and the support of user interaction.

The system is part of the MODE project (Multimedia Objects in a Distributed Environment) [Bla90, Bla91] that is used in NESTOR (Networked Systems for TutORing) [Mue91], an authoring-learning environment that is in development at the 
Universities of Karlsruhe and Kaiserslautern in cooperation with the CEC Karlsruhe, Digital Equipment.

The overall synchronization system in MODE consists of four components:

- the Synchronization Editor that is used to create synchronization and layout specifications for multimedia presentations;

- the Global Synchronization Coordinator that coordinates the creation of presentation units (presentation objects) from the basic information units (information objects) and the transport of the objects in the distributed system;

- the Synchronizer that receives the presentation objects from the Global Synchronization Coordinator and initiates their local presentation according to the synchronization specification;

- the Optimizer that chooses presentation qualities and presentation forms depending on user demands, network and workstation capabilities and presentation performance.

In the following, after an overview about the related work, our synchronization model, the Synchronizer and the Synchronization Editor are described in more detail.

\section{Related Work}

Synchronized multimedia presentations, called multimedia objects, are composed of singlemedia objects, multimedia objects and synchronization information. This allows to create synchronization hierarchies. Examples for single-media objects are a video sequence, a piece of text or a picture.

Several ways of describing multimedia synchronization have been published:

Hierarchical synchronization: Multimedia objects are regarded as a tree consisting of nodes that denote serial or parallel presentation of the outgoing subtrees.

Synchronization on a time axis: Single-media objects are attached to a time axis that represents an abstraction of time.

Synchronization at reference points: Single-media presentations are composed of subunits presented at periodic times. A position of a subunit in an object is called a reference point. Synchronization between objects is defined by connections between subunits of different objects that has to be presented at the same time.

Synchronization described by means of hierarchical structures [AFN89, SS89] is based on the two main synchronization operations:

- Serial synchronization of actions

- Parallel synchronization of actions 
An action is either an atomic action or a compound action. An atomic action handles a presentation of a single-media object, a user input or a delay. Compound actions are a combination of synchronization operators and atomic actions.

The introduction of a delay as possible action [LG90] allows to model further synchronization behavior like delays in serial presentations and delayed presentation of a single object in a parallel synchronization.

Hierarchical structures are easy to handle and are widely used. Restrictions from the hierarchical structure arise by the fact that each action can only be synchronized at its beginning or end. For example presenting a subtitle at a certain scene of a video stream requires dividing the video stream into serial components. In the same way a synchronized multimedia object used as a component in another synchronization can not longer be regarded as abstract unit, if it has to be synchronized at a point between beginning and end of its presentation. Therefore abstractions from the internal structure of multimedia objects cannot be achieved in general. Additionally, synchronization conditions exist that cannot be represented in hierarchical structures. An example are three objects that are presented in parallel and each object is synchronized once with each other object but independent of the third object.

Besides hierarchical structures synchronization can also be defined by means of a time axis. In the Athena Muse project [HSA89] a synchronization is described by attaching all objects independent of each other to a time axis. Removing one object does not effect the synchronization of the other objects.

With modifications this kind of specification is used in the model of active media [TGD91]. A world time is maintained which is accessible to all objects. Each object can map this world time to its local time and moves along its local time axis. If it detects a discrepancy between world time and local time exceeding a limit it has to synchronize to world time again.

A time axis based mechanism is used in QuickTime [LM91], too.

Synchronizing objects by means of a time axis offers the opportunity of abstractions from the internal structure of single-media objects and multimedia objects used in further synchronizations. Defining the beginning of a presentation of a subtitle relative to a scene in a video stream requires no knowledge about the related video frames. Problems arise if objects have no deterministic time of presentation (like presentations of objects depending on user interactions) because synchronization can only be defined using fixed points of time and if synchronization based on one common world time is not sufficient to express the synchronization conditions between presentation streams. Depending on the coherence of the presentation streams a synchronization by using a common time axis might be to strong or to weak.

A third way of defining synchronization is a description of timing relations between objects without explicitly referencing time. Steinmetz [Ste90] proposes such a model. Dynamic objects such as video or audio are regarded as composed of periodic presentations of subunits for examples video frames or audio samples. In this case synchronization is specified by denoting subunits in objects that shall be presented at the same moment.

Like synchronizing by means of a time axis this description allows synchronization not only at the beginning or end of presentations but also during a presentation. Synchro- 
nizing at reference points requires in contrast to hierarchical structures mechanisms for detecting inconsistencies. It is impossible to create delays during a multimedia presentation using only reference points of objects. To solve this problem Steinmetz proposes time specifications. A time specification introduces a delay at a certain point of synchronization.

We are using a synchronization model based on synchronization at reference points extended by means of handling fixed intervals of time, objects of unpredictable duration and conditions resulting from the underlying distributed heterogeneous environment.

\section{Synchronization Model}

MODE allows to give classes one or more of the attributes information class, presentation class and transport class. Objects of information classes (information objects) are the basic information units. If information objects should be presented, they generate one or more objects (presentation objects) of suitable presentation classes. Presentation objects contain all data and methods to display themselves and can exist independently from the information object. Especially they give the choice to transport the basic information unit or to transport its presentation in the case of a remote presentation. If an information or a presentation object has to be transported, it is converted into an object of a transport class (transport object) for the duration of the transport and on the other node it is reconverted. Transport objects consist of all methods and data necessary to transport and compress the object.

The generation, display and conversion/reconversion operations are initiated by calling corresponding methods on the objects. Additionally the information objects offer methods that give information about available presentation forms or timing behavior of presentation objects. Using these method interfaces of the information and presentation objects, the Synchronization Editor and the Synchronizer can handle any kind of user-implemented media.

A synchronization specification created with the Synchronization Editor and used by the Synchronizer is stored in text form following a syntax defined in a context free grammar (Synchronization Description Language). This allows usage of the synchronization specification by MODE components independent of their implementation language and environment.

An overview about the architecture is shown in figure 1 .

Information objects may support various presentation forms and qualities. Presentation forms supported by a text information class may be a presentation as text on the screen or as audio sequence generated by a speech synthesizer. Presentation qualities of a video sequence are color depth, resolution and frames per seconds. If an information object is used in a multimedia object its properties have to be determined more precisely. In addition an information object should not be influenced by its utilization in a multimedia object. Therefore basic objects are introduced. A basic object consists of a reference to an information object and a collection of presentation attributes.

We distinct between dynamic basic objects and static basic objects. A presentation of a dynamic basic object is composed of a sequence of presentation objects (for example 
single frames of a video sequence) presented at periodic times. The synchronization of such a single-media sequence is called intra-object synchronization. The index of each presentation object is called a reference point. A presentation of a static basic object such as displaying a piece of text or a picture has only two reference points, the beginning and the end of the presentation.

The synchronization between presentations of basic objects (inter-object synchronization) is defined using reference points. A synchronization element is a combination of a reference point and the corresponding basic object (BasicObject.ReferencePoint). It denotes a position in an information object presentation. Two ore more synchronization elements are defining a synchronization point that is the base of the inter-object synchronization. The list of all synchronization points specifies the whole inter-object synchronization.

A reference point may be a natural number, referencing for example the number of a video frame, or a fraction between 0 and 1 that we call a relative reference point. Before presentation, relative reference points are mapped to their nearest non-relative reference point. Since multimedia objects have no obvious external reference points they may only be synchronized relatively.

Inter-object synchronization at reference points has the following advantages:

- It is possible to choose between loosely and tightly coupled synchronization of presentations of dynamic objects. This is much more flexible than simply using one absolute or virtual timer as common synchronization base.

- The synchronization of objects with non-predictable presentation duration can be handled easily.

- The synchronization points can be maintained if presentation objects are delayed.

- Useful manipulations such as fast-forwarding, rewinding or slow-motion can simply be realized by changing the periodicity of the presentations of the dynamic objects. This does not effect the synchronization points a multimedia presentation consists of.

Two special kinds of objects are supported additionally, timers and interactive objects.

Timers are objects with the ability to synchronize at reference points that occur in $1 \mathrm{~ms}$ steps. Timers are used to model absolute delays like in a slide-show where every $10 \mathrm{~s}$ the slide changes. Also the synchronization based on one common time axis can be modeled in our synchronization schema by using one timer.

Interactive objects are objects synchronized only at beginning and end that have the additional ability to communicate with the user. They are examples for objects of nonpredictable presentation duration. Depending on user interactions interactive objects can for example use methods offered by the Synchronizer. These methods cause a starting or stopping of a presentation of a basic object, simulate fast-forwarding or rewinding of multimedia objects, or move the presentation to a certain point in a multimedia object. For every basic or multimedia object that is part of a multimedia object one or more alternative presentations can be specified that are used if the preferred presentation can not be used due to limitations of the workstation or network resources. If a workstation 
has for example no audio device an alternative presentation form might be subtitles. Each alternative may add additional synchronization elements that replace those of the preferred object.

For each basic object a presentation quality can be specified. The presentation quality is described by a set of attributes consisting of an attribute name, a preferred value, a value domain that describes all possible values for this attribute and a priority. Before starting a presentation the Optimizer is called that chooses a value from the domain according to user demands and the capacity. of network and workstation resources. If possible, the preferred attribute value is chosen. The priority of an attribute describes the importance of its attribute value relative to other attributes.

Three types of actions are used to define a behavior in exception conditions occurring during a synchronized presentation.

Waiting action: A presentation of a dynamic basic object has reached a synchronization point and waits longer than a specified time at this synchronization point. Possible actions are displaying the last presentation object, pausing or ignoring the synchronization point.

Acceleration action: A presentation of a dynamic basic object has reached a synchronization point. Acceleration actions are necessary for other presentations of dynamic basic objects, if they are involved in this synchronization point and will need longer than a specified time to reach it. Possible actions are to temporarily increment the presentation speed or to skip all objects in the presentation up to the synchronization point and to continue there immediately.

Skipping action: A presentation object to present next has not arrived in time. A possible action might be to skip the object and to present the next one.

A basic object may have a priority that indicates its sensitivity to delays of its presentation. For example, audio objects will in general be assigned higher priorities than video objects because a user recognizes delays in an audio-stream earlier than those in a video-stream. Presentations with higher priorities are preferred in presentation and synchronization to objects with lower priorities.

Depending on the timing behavior during a presentation, a graceful degradation or enhancement of presentation quality is possible.

Using synchronization at reference points causes problems if parts of an information object that are used in multimedia objects are changed. Synchronization is lost, for example, if a scene in a video object is inserted or removed. Therefore a kind of version controlling is necessary that allows mapping synchronization from one version to another.

\section{The Synchronizer}

The task of the Synchronizer [Hue91] is to perform the synchronized presentation (figure 2) according to the introduced synchronization model. This comprises the intra-object and the inter-object synchronization. 
For each intra-object synchronization a presentation thread is created managing the presentation of a dynamic basic object. The priorities of these threads are used to implement priorities of basic objects. All presentations of static basic objects are managed by a single thread.

Synchronization is performed by a signaling mechanism. Each presentation thread reaching a synchronization point sends a corresponding signal to all other presentation threads involved in the synchronization point. After having received such a signal other presentation threads may perform acceleration actions, if necessary. After having sent all signals the presentation thread waits until it receives all signals from the other participating threads of the synchronization point and may perform a waiting action.

The presentation threads are also responsible for executing skipping actions.

The Synchronizer also supports joining of different basic objects. Joining is useful for example to reduce transport costs, if bitmaps for video frames and subtitles are created on the same node. In such a case they may be joined at the node of creation to a single bitmap containing video and text information.

\section{The Synchronization Editor}

A specification of a synchronization in a textual form is difficult. In text-oriented languages it is not easy to imagine the timing and layout relations between the objects. The graphical Synchronization Editor [Lan91] eases the specification of synchronization and reduces the time for creating multimedia objects.

Describing multimedia objects the user has to regard three dimensions. Beside the two spatial dimensions that are used in most applications only dealing with static media, the time has to be regarded as third dimension.

Editing a three-dimensional view [OHK90] on the screen raises some problems. The position of three-dimensional elements on two-dimensional input and output devices is not unique. Since multimedia objects consist of a collection of coherent objects, many objects will be hidden by others. To avoid these problems we use three types of twodimensional views of a multimedia object.

In the first kind of view, the Presentation View (figure 3), the user can run single objects independent of each other. Similar to a tape recorder he can stop, pause, restart, fastforward and rewind them. This presentation is supported by the Synchronizer. By viewing the objects of the synchronization it is easy to find suitable synchronization points. A synchronization point between all currently presented objects is created by clicking on a button. In the same way the user can switch to other synchronization points. To get a quick impression of the edited multimedia object the user can regard it in the same way as the single objects.

For visualizing and directly editing the timing relations between the objects the second view - the Time View (figures 4 and 5) - is used. It shows all objects ordered at a horizontal time axis. Objects are represented by rectangles. Their width indicates their expected duration. A synchronization point is denoted by a vertical line between the objects. Editing facilities comprise adding, moving, deleting of synchronization points and basic objects and selection of points of time in the synchronization. 
Since not all objects are presented during the whole representation, the layout of the multimedia object changes in time. That is why the layout cannot be regarded independently of the synchronization. A third view - the Layout View (figure 6) - is implemented in order to edit the layout at a certain point of time in the synchronization. It uses a layout model [Emm91] that allows to specify absolute and relative distances that are needed if the presentation layout is resized at runtime.

More instances of each type of view can be opened in parallel, for example several open Layout Views may show snapshots at different points of time.

\section{Summary}

Tools for specifying and executing multimedia presentations have been presented. The Synchronization Editor allows the user to graphically specify the synchronization of multimedia objects. The actual local presentation is done by the Synchronizer that initiates and coordinates the presentations.

The Synchronizer cooperates with the Optimizer and the Global Synchronization Coordinator that is responsible for the transport of the presentation units.

A standardized interface to the media objects enables the tools to integrate any kind of user-defined media.

The object-oriented synchronization model that is used by the tools is an extension of the synchronization at reference points and allows the integration of objects of unpredictable duration, user interactions and timers.

Designing the synchronization system, we have taken care of the difficulties in presenting multimedia objects raising from the underlying distributed heterogeneous environment. These difficulties are different capacities of the available resources and possible errors like network delays and loss of presentation, units.

Therefore, on specifying the synchronization the user can determine alternative presentations to objects, that will be used if the first choice is impossible because of resource conditions. The user can also specify waiting, acceleration and skipping actions to handle delayed or lost presentation units.

The synchronizer uses the presentation form and quality, that is selected by the Optimizer regarding the existing resources and user demands. If an exception condition occurs at runtime the synchronizer performs the specified actions.

Our practical experiences have shown the usefulness of the presented schema. Further work is on the full integration with the Global Synchronization Coordinator and the Optimizer as well as an implementation based on realtime scheduling.

\section{References}

[AFN89] AFNOR Expert group, Multimedia synchronization: definitions and model, Input contribution on time variant aspects and synchronization in ODAextensions, ISO IE JTC 1/SC 18/WG9, February 1989. 
[Bla90] Gerold Blakowski, "Supporting Multimedia Information Presentation in a Distributed, Heterogeneous Environment", in Proceedings 2nd IEEE Workshop on Future Trends of Distributed Computing Systems, Cairo, Egypt, September 30 - October 2, 1990, pages 29-35, Los Alamitos: IEEE Computer Society Press 1990.

[Bla91] Gerold Blakowski, "The MODE-FLOW-GRAPH: A Processing Model for Objects of Distributed Multimedia Applications", to be published in Proceedings International Symposium on Communication, Tainan, Taiwan, December 1991.

[Emm91] Richard Emmerich, "Design and Implementation of a Geometry Manager in X-Windows". Diploma thesis (in German), University of Karlsruhe, Institute for Telematics, Germany, April 1991.

[HSA89] Matthew E. Hodges, Russell M. Sasnett, and Mark S. Ackerman, "Athena Muse: A Construction Set for Multi-media Applications", IEEE Software, pages 3743, January 1989.

[Hue91] Jens Hübel, "Design and Realization of a Runtime System for the Synchronized Presentation of Multimedia Data Streams". Diploma thesis (in German), University of Karlsruhe, Institute for Telematics, Germany, September 1991.

[Lan91] Ulrike Langrehr, "Design and Realization of a Graphical User Interface for the Definition of the Synchronized Presentation of Multimedia Data Streams". Diploma thesis (in German), University of Karlsruhe, Institute for Telematics, Germany, September 1991.

[LG90] Thomas D. C. Little and Arif Ghafoor, "Synchronization and Storage Models for Multimedia Objects", IEEE Journal on Selected Areas in Communications, 8(3):413-426, April 1990.

[LM91] D. Littman and T. Moran, "Quicktime: It's about Time", Mac World, pages 80-81, August 1991.

[Mue91] Max Mühlhäuser, "Computer Based Learning with Distributed Multimedia Systems", in Hans Jürg Bullinger, editor, Human Aspects in Computing, Proceedings of the IVth International Conference on Human Computer Interaction, Stuttgart, Germany, September 1-6, 1991, Volume 2, pages 953-958, Amsterdam: Elsevier 1991.

[OHK90] Ryuichi Ogawa, Hiroaki Harada, and Asao Kaneko, "Scenario-based Hypermedia: A Model and a System", in Hypertext: Concepts, Systems and Applications, Proceedings of the European Conference on Hypertext INRIA, France, November 1990, pages 39-51, 1990.

[SS89] Michael Salmony and Doug Shepherd, "Extending OSI to Support Synchronization Required by Multimedia Applications", Technical Report 43.8904, IBM European Networking Center, Tiergartenstr. 8, 6900 Heidelberg, P.O.Box 103068, Germany, April 1989.

[Ste90] Ralf Steinmetz, "Synchronization Properties in Multimedia Systems", IEEE Journal on Selected Areas in Communications, 8(3):401-412, April 1990.

[TGD91] Dennis Tsichritzis, Simon Gibbs, and Laurant Dami, "Active Media", in Dennis Tsichritzis, editor, Object Composition, pages 115-132, Genève: Université de Genève, Centre Universitaire d'Informatique 1991. 


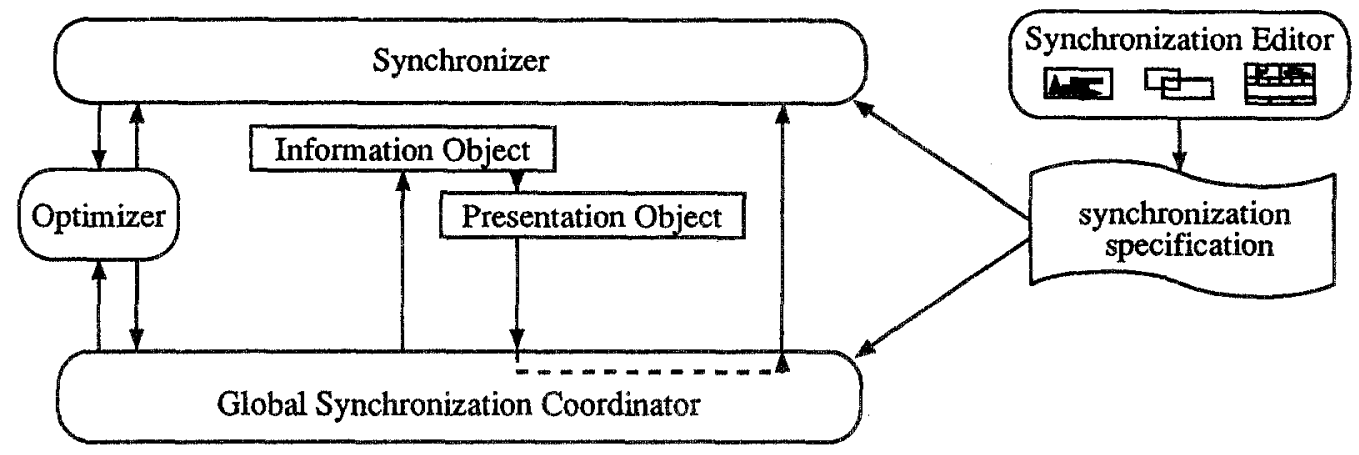

Figure 1: The architecture of the synchronization components of MODE.
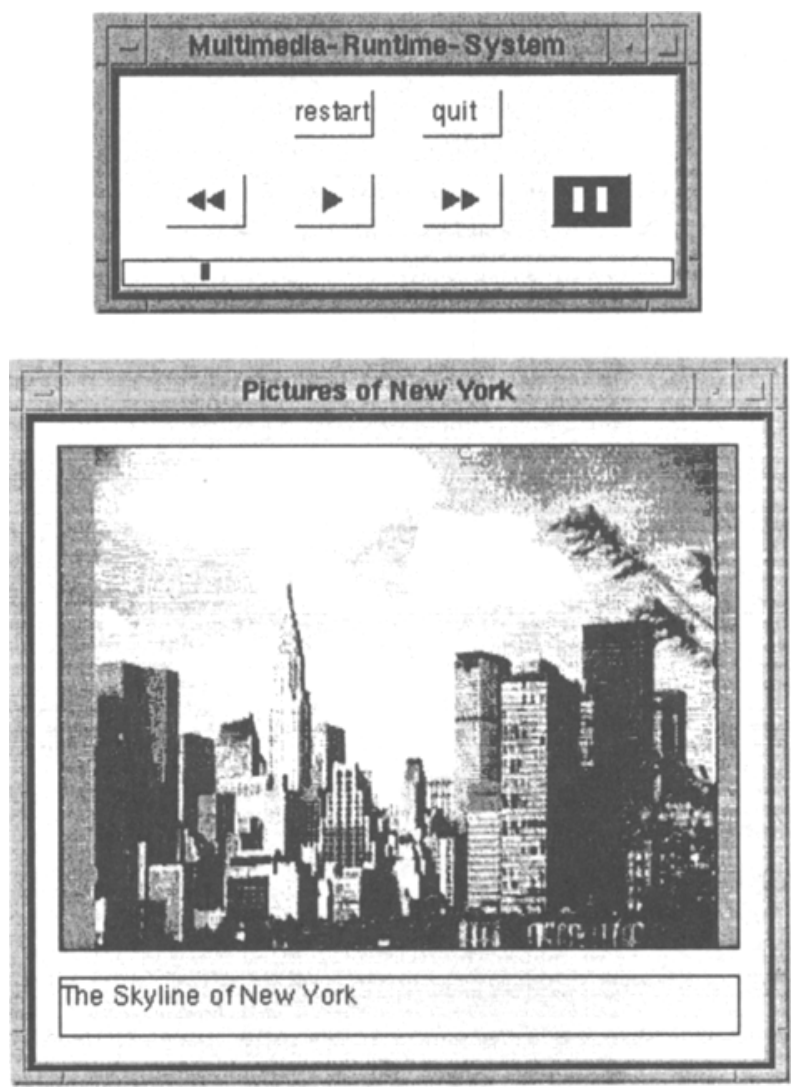

Figure 2: The output of the Synchronizer for a subtitled video presentation created with the Synchronization Editor. 


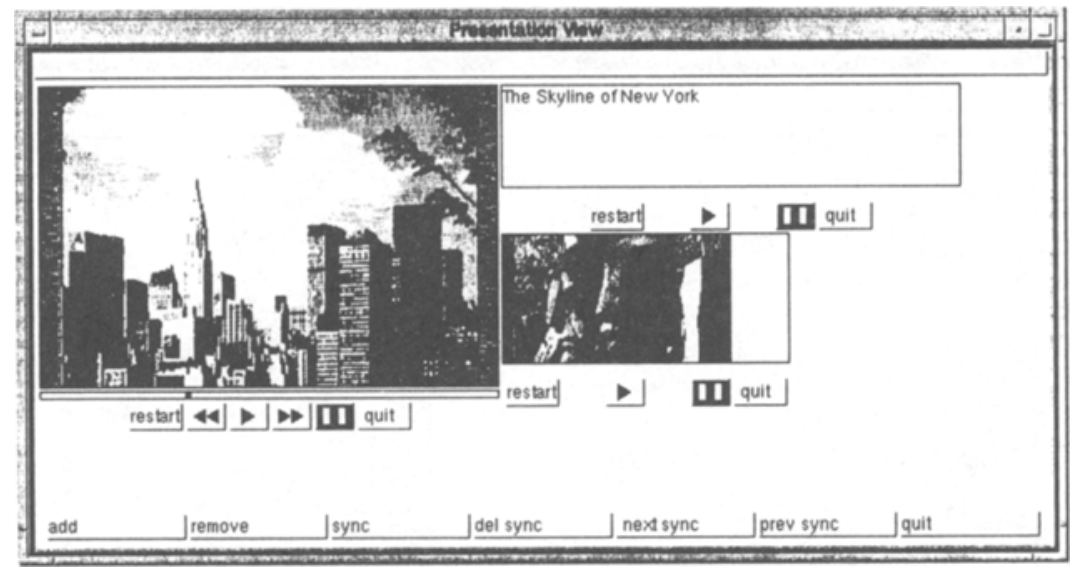

Figure 3: The Presentation View allows to display single objects to find suitable synchronization points.

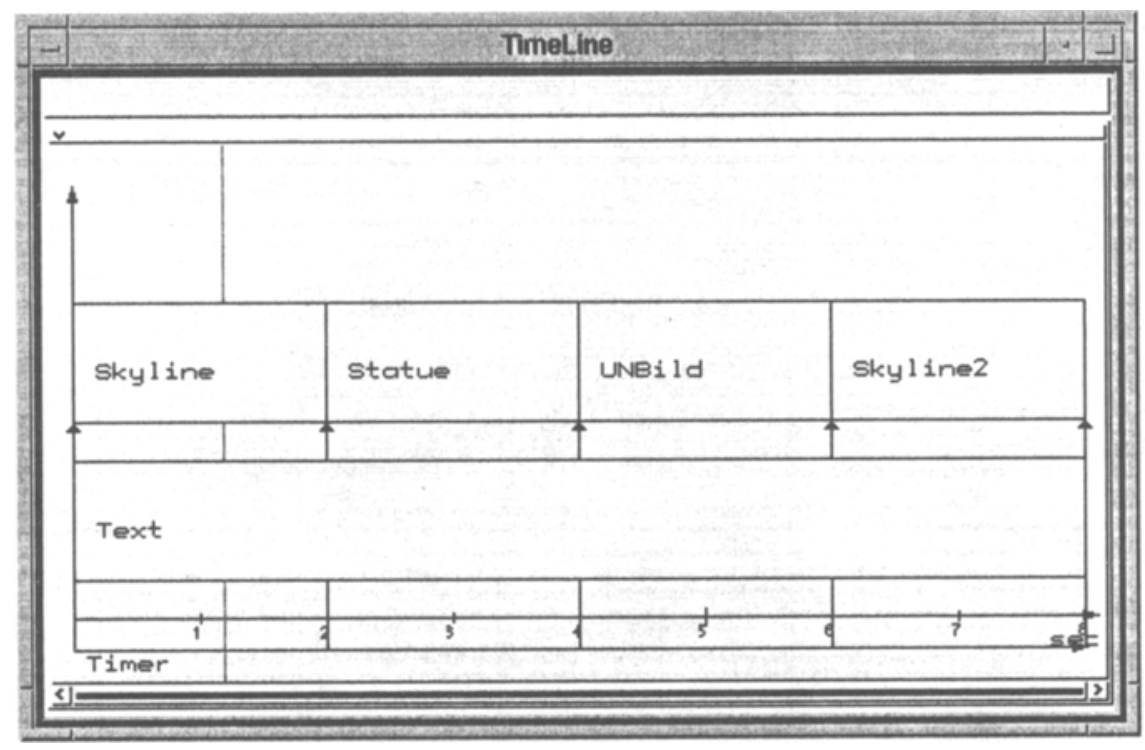

Figure 4: This Time View shows how a slide show is synchronized using a timer object. 


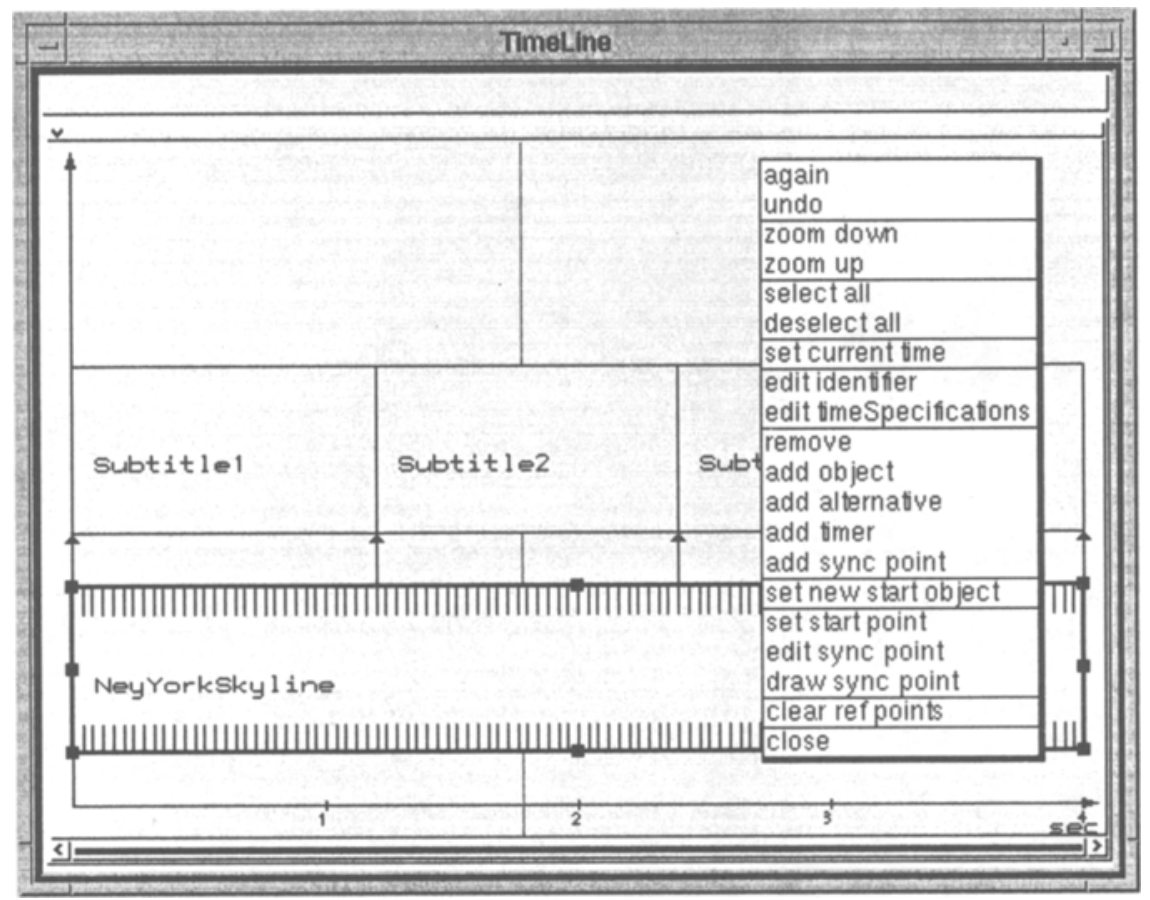

Figure 5: This Time View shows the synchronization of a video with subtitles and a popup menu with some editing options.
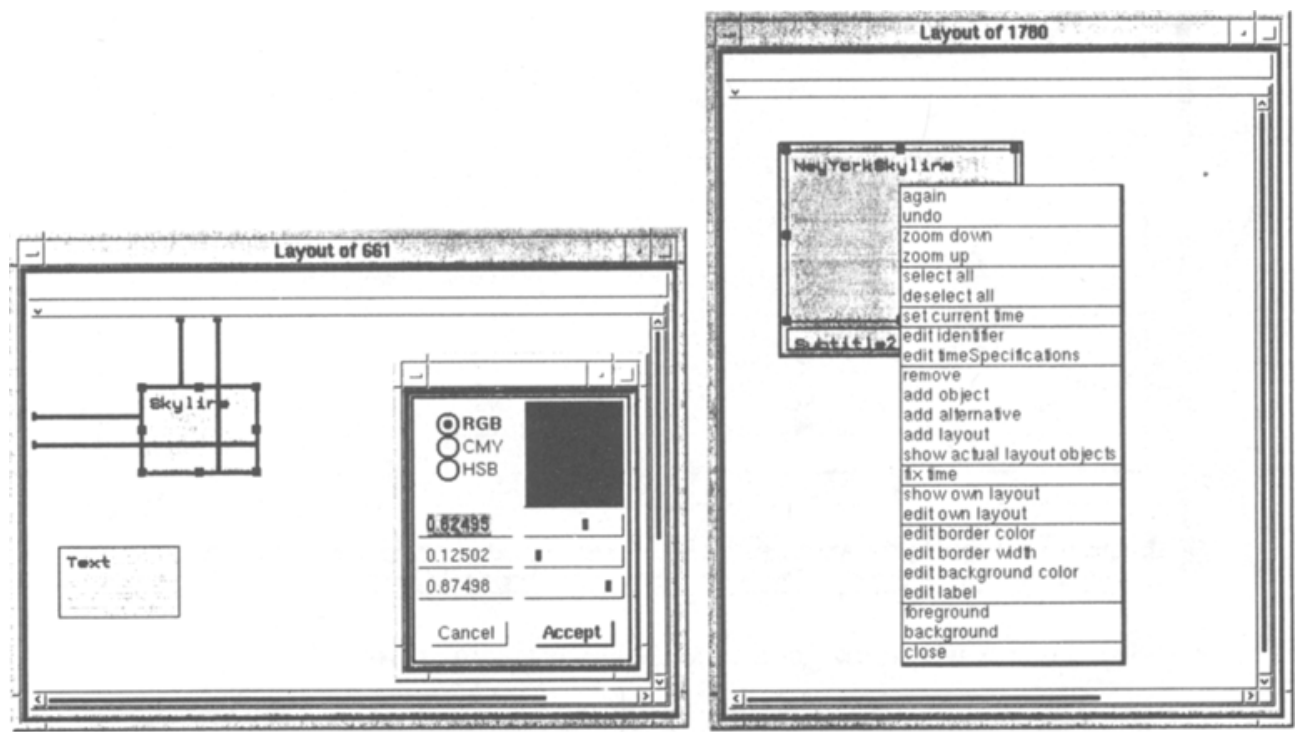

Figure 6: The left Layout View shows the layout of the slide show at a time selected in the Time View and how for example a border color may be specified. The right Layout View shows a video and a subtitle that are grouped to be presented in a single window. 